\title{
Negative und positive Verstärkung bei pathologischem Glücksspielen: Ihre mögliche Bedeutung für die Theorie und Therapie von Zwangsspektrumsstörungen
}

\author{
I. Hand \\ Universitätsklinikum Eppendorf, Hamburg, Deutschland
}

\section{Schlüsselwörter}

Glücksspielen · Verstärkung · Zwangsspektrumsstörungen ·

Nichtstoffgebundene Süchte $\cdot$ Verhaltenstherapie

\section{Zusammenfassung}

Eskaliertes "Normalverhalten" oder Verhaltensexzesse werden unter bestimmten Bedingungen als pathologisch, also als behandlungsbedürftige Störungen definiert. Waschen, Laufen, Kaufen, Glücksspielen oder Alkohol trinken im schädlichen Übermaß werden teilweise den Zwangs(spektrums)störungen, teilweise den Süchten zugeordnet. Manche Autoren benutzen die Diagnosen Sucht- und Zwangsstörung auch synonym. Verbreitet ist die Dreiteilung in Zwangsstörungen, Zwangsspektrumsstörungen oder nichtstoffgebundene Abhängigkeiten/ Süchte und Suchterkrankungen. Der Dissens "Zwang oder Sucht?» ist auf die mittlere Gruppe beschränkt.

Am Beispiel des pathologischen Glücksspielens wird der Versuch unternommen, therapie- und forschungsrelevante Hypothesen am "roten Faden" negativer und/oder positiver Verstärkung abzuleiten. Dabei wird auf die Entwicklung der beiden Hauptstörungsmodelle - Sucht oder Zwang(sneurose) - in den letzten 40 Jahren Bezug genommen. Unterschiede innerhalb der verhaltenstherapeutischen Ansätze einerseits und Gemeinsamkeiten zwischen multimodalen verhaltenstherapeutischen und tiefenpsychologisch-analytischen Modellen andererseits werden verdeutlicht.

Ausgehend von den Verstärkerfunktionen werden Indikationskriterien sowohl für "symptom-» oder "ursachenbezogene» Verhaltenstherapie (oder deren Kombination) als auch für die Pharmakotherapie, vorgeschlagen. Anwendungsbeobachtungsstudien legen die teilweise Übertragung dieses Modells auf andere Zwangsspektrumsstörungen nahe. Dieser Beitrag setzt dabei schwerpunktmäßig Akzente zur Beschreibung der Subgruppe pathologischer Glücksspieler, für die dieses Modell entwickelt wurde; er vereinfacht damit unvermeidlich die Komplexität pathologischen Glücksspielens. Der gesamte Bereich der Zwangsspektrumsstörungen befindet sich noch im Stadium der Hypothesen- und Modellbildung und daraus abgeleiteter Anwendungsbeobachtungsstudien. Aufgrund zunehmender Nachfrage besteht erheblicher Handlungsbedarf für Therapeuten und Therapieforscher.

Dieser Artikel ist nicht als Beitrag zur Modelldiskussion "Sucht» bei den stoffgebundenen Störungen gedacht.

\author{
Key Words \\ Pathological Gambling · Reinforcement . \\ Obsessive compulsive spectrum disorders . \\ Drug-independent addictions · Behavior Therapy
}

\section{Summary}

Negative and Positive Reinforcement in Pathological

Gambling: Their Potential Impact on Theory and Therapy of

Obsessive-Compulsive Spectrum Disorders

Everyday 'normal' behaviors that escalate ('behavioral excesses') are considered pathological, if certain criteria are fulfilled. Washing, running, shopping, gambling or drinking too much alcohol are sometimes labeled obsessive-compulsive (spectrum) disorders, sometimes labeled addictions. Some authors use both diagnoses synonymously. It is also quite common to differentiate between obsessive-compulsive disorders, obsessivecompulsive spectrum disorders or drug-independent addictions, and addictions. The dispute is limited to the middle group.

Taking pathological gambling as an example, we will try to derive a treatment and research relevant hypothetical model of gambling on the basis of negative and/or positive reinforcement as diagnostic guideline. Reference is made to the development of the two main treatment models of pathological gambling - addiction or obsessive-compulsive (neurotic) disorder over the past 40 years. The new multimodal, 'causal' behavioral treatment model is compared to symptom-oriented behavioral treatments on the one hand and 'causal' psychoanalytic approaches (including recent addiction theories) on the other hand.

Criteria for the indication of 'symptom' or 'cause' oriented behavioral treatments (or combinations thereof) and for drug treatments are derived from the reinforcement model. The application of this model to other obsessive-compulsive spectrum disorders is suggested, referring to first clinical observational studies. The article focuses on main aspects of pathological gambling to describe the subgroup of patients for whom this model was developed; therefore, it necessarily simplifies the complexity of problem gambling. The field of obsessive-compulsive spectrum disorders is still in a stage of development of hypotheses and models, relying on rather few clinical observational studies. Yet, the demand for treatment is increasing and urges therapists and researchers to respond.

This article is not meant to be a contribution to the discussion of models of drug-related problems and disorders.

\begin{tabular}{ll}
\hline KARGER & @ 2004 S. Karger GmbH, Freiburg \\
Fax +49 7614520714 & Accessible online at: \\
$\begin{array}{l}\text { E-mail Information@Karger.de } \\
\text { www.karger.com }\end{array}$ & www.karger.com/ver
\end{tabular}




\section{Einleitung}

Wenn normale Verhaltensweisen wie Waschen, Kaufen, Arbeiten, Sexualität oder auch Glücksspielen oder Alkohol trinken so sehr eskalieren, dass sie den Umgang des Individuums mit sich selbst oder seinem sozialen Umfeld empfindlich beeinträchtigen, dann wird von einer «Störung» im Sinne der diagnostischen Klassifikationssysteme DSM und ICD gesprochen. Wie eine solche Störung aber zu bezeichnen, ätiologisch $\mathrm{zu}$ verstehen und folglich zu behandeln ist, darüber gehen die Meinungen seit Jahrzehnten auseinander. Die am häufigsten verwendeten, eher beschreibenden Begriffe sind: Verhaltensexzesse [z.B. Hand, 2002a] bzw. Behavioral Excess Disorders [z.B. Shaffer, 2002], Excessive Appetites [Orford, 1985], problematisches Verhalten [Hand und Kaunisto, 1984] bzw. Problem Behaviors [Petry, 2002b], Selbstkontrollproblem, auch für stoffgebundene Süchte [z.B. Brister und Brister, 1987; Baumeister et al., 1994]. Bei diesen Begriffen bleibt oft unklar, ob sie pathologische Verhaltensweisen im Sinne der Klassifikationssysteme bezeichnen sollen oder eine Verhaltenseskalation zwischen «normal» und «pathologisch». Was die Zuordnung der Verhaltensexzesse zu den Süchten angeht, reichen die Positionen von «wir leben in einer versüchtelnden Gesellschaft»[Gross, 1990] bis «Süchte gibt es nicht» [Davies, 1997].

Auf der deskriptiv-diagnostischen Ebene von ICD und DSM werden einige Verhaltensexzesse unter «Impulskontrollstörungen» zusammengefasst. In diesem Rahmen werden sie dann oft als «pathologisch» bezeichnet (z.B. pathologisches Glücksspielen).

In der ätiologisch orientierten Diagnostik geht die Diskussion seit Jahrzehnten um die Frage, ob Verhaltensexzesse Süchte, also «nichtstoffgebundene Abhängigkeiten» (psychische Abhängigkeit als Sucht in Reinform, ohne Konfundierung durch bewusstseinsverändernde Stoffe [z.B. Orford, 1985; Gross, 1990]), oder Zwangsstörungen im Sinne von Zwangsspektrumsstörungen [Hollander, 1993] darstellen - und damit Neurosen im Sinne der Klassifikation bis zu DSM-III und ICD-10. Es wird schließlich bis heute auch immer wieder diskutiert, ob Süchte und Zwangsstörungen nicht das Gleiche sind [z.B. Dodes, 1996; Vent, 1999]. Die meisten Autoren gingen in der Vergangenheit davon aus, dass eine Achse-I-Diagnose eine homogene Patientenpopulation beschreibt - auch in den $\mathrm{Pu}$ blikationen zu pathologischem Glücksspielen [Blanco et al., 2001]. Entsprechend ging der Disput auch darum, welches die «richtige» ätiologische Zuordnung und deren therapeutische Konsequenzen seien. Seit einigen Jahren setzt sich nun die Erkenntnis durch, dass Achse-I-Diagnosen ätiologisch sehr unterschiedliche Subgruppen zusammenfassen und daher unterschiedliche Interventionen benötigen [z.B. Blanco et al., 2001].

Die diagnostische Zuordnung pathologischen Glückspielens zu nichtstoffgebundenen Abhängigkeiten oder Zwangsspektrumsstörungen hatte in den 80er Jahren gravierende Konse- quenzen für Betroffene: Im einen Fall erhielten sie klassische Suchttherapie, im anderen Psychotherapie (s.u.). In den vergangenen 2 Jahrzehnten haben die Versorgung und die Versorgungsforschung im Bereich der Verhaltensexzesse auf der einen und der stoffgebundenen Süchte auf der anderen Seite zu einer tiefgehenden Veränderung der Diskussion über das «Wesen» von Sucht (z.B. psychische Abhängigkeit als Kernelement von Sucht) und deren effektivste Behandlung geführt. Dies gilt besonders für den Vergleich der Merkmale pathologischer Glücksspieler und alkohol- oder drogenabhängiger Personen. Während in den 80er Jahren von Seiten der Suchttherapeuten das Abstinenzgebot aus der Behandlung stoffgebundener Süchte vielerorts ohne Modifikation auf pathologisches Glücksspielen übertragen wurde, entwickelte sich international aus den Arbeiten einiger Behandlungszentren für pathologische Glücksspieler und auch aus der Forschung über stoffgebundene Süchte eine Relativierung des Abstinenzgebotes. Hierzu haben insbesondere die Konzepte des «Vermeidungsspielers» [Lesieur, 1988] und der «Selbstmedikationshypothese» [Khantzian, 1985, 1997] bei stoffgebundenen Süchten beigetragen. Die Selbstmedikationshypothese wurde später auch auf pathologisches Glücksspielen übertragen [Khantzian, 2002]. In beiden Konzepten wird das Problemverhalten als subjektiver Problemlöseversuch für zugrunde liegende, vorher bestehende Störungen interpretiert. Die früher teilweise apodiktischen Forderungen nach «Erziehungsmaßnahmen» für Personen mit Suchtproblemen weichen zunehmend der Erkenntnis, dass bei vielen Betroffenen erst einmal die Motivation geklärt und modifiziert werden muss, bevor eine gemeinsame therapeutische Basis gefunden werden kann [Miller und Rollnick, 1991]. Erst dann können eine alternative Selbstmedikation bzw. Selbsthilfe als Ersatz für das Problemverhalten angestrebt werden.

Bezüglich der zahlreichen, detaillierteren ätiologischen Modelle zum Glücksspielen muss im Rahmen dieses Artikels auf andere Darstellungen verwiesen werden [z.B. Klepsch et al., 1989b; Wildman II, 1997; Petry, 2003; Meyer und Bachmann, 2000; O'Connor et al., 2004]. International vorherrschend sind immer noch die zwei klassischen Modelle pathologischen Glücksspielens: stoffungebundene Sucht oder Zwangsspektrumsstörung; die endgültige nosologische Zuordnung ist noch nicht absehbar [Blanco et al., 2001]. Letztere Autoren halten aufgrund einer aktuellen Literaturübersicht folgende Untergruppen für denkbar: 1. eher Suchtkranken ähnlich; 2. eher Zwangskranken ähnlich; 3. mit Merkmalen von Sucht- wie Zwangskranken.

In diesem Artikel wird eingangs die «historische» Entwicklung der Theorie- und Therapiekonzepte bei Verhaltensexzessen am Beispiel der «Suchttherapie» und der «Neurosentherapie» pathologischen Glücksspielens nachgezeichnet. Es folgt ein Überblick über wesentliche aktuelle Entwicklungen. Dabei wird deutlich, dass der bisherige Publikationsstand eher klinisch-empirisch gestützte Hypothesen als evidenzbasierte 
Erkenntnisse darstellt [O'Connor et al., 2004]. Abschließend wird eine zusammenfassende, prospektive Hypothesenbildung vorgestellt. Als «roter Faden» wird die Funktionsdiagnostik des (pathologischen) Glücksspielens auf der Ebene positiver und negativer Verstärkung gewählt. Mit Hilfe der Zuordnung klinischer Hypothesen in ein Modell negativer und/oder positiver Verstärkung wird eine überprüfbare, grundsätzliche (Be-)Handlungs- und Indikationsstrategie vorgestellt, bei der das Verhalten an sich hinter seiner Funktion deutlich zurücktritt. Mögliche Parallelen zwischen dem hier vorgestellten Glücksspieler-Modell und anderen Verhaltensexzessen werden angedeutet.

\section{Die "historische» Entwicklung wesentlicher Modell- vorstellungen zum pathologischen Glücksspielen}

\section{Suchtmodell oder Neurosemodell? Die Diskussion in Deutschland}

Anfang der 1980er Jahre wurde pathologisches Glücksspielen als bedeutsames Versorgungsproblem in Deutschland auffällig. In der Wirtschaftskrise Mitte der 80er Jahre begann ein Boom von Spielhallen mit «Unterhaltungsautomaten mit Gewinnmöglichkeit». Frustration, Schamgefühl oder Depression führten manchen Arbeitslosen als «Vermeidungsspieler» dorthin. Solche Personen machten damals - nach anhaltenden Spielverlusten - zeitweise über $50 \%$ der in der Hamburger Verhaltenstherapie-Ambulanz Hilfe suchenden Glücksspieler aus [Hand und Kaunisto, 1984].

Ab Mitte der 80er Jahre boten auch Einrichtungen der Suchtkrankenversorgung Hilfe für Glücksspieler an. Deren Suchttherapie war am Störungsmodell der Gamblers Anonymous (GA) orientiert und sah lebenslange Abstinenz als entscheidendes Ziel. In dieser Zeit entstand eine heftige Diskussion darüber, ob das pathologische Glücksspielen eine Sucht oder eine Neurose sei [vgl. Hand, 1986].

Im klassischen Suchtmodell triggern Kontakte mit einer Glücksspielsituation (möglichst mit deutlichen Gewinnen) eine in der Person angelegte, aber noch nicht manifeste Glücksspielsucht, aus der es danach kein Zurück in den $\mathrm{Zu}$ stand der «Unschuld» mehr gibt. Primäres Therapieziel ist lebenslange Abstinenz durch tägliche Impulsunterdrückung oder -kontrolle. Bei dessen Einleitung sind Entzugssymptome das Hauptrückfallrisiko. Als Entzugssymptome wurden vor allem Unlust, Reizbarkeit, Schlafstörungen, Alpträume, Schweißausbrüche, Zittern, motorische Unruhe und innere Unruhe angeführt [vgl. Meyer und Bachmann, 2000]. Custer und Milt hatten aber schon 1985 festgestellt, dass Entzugssymptome nichts mit Glücksspielsucht zu tun hätten. Tavares et al. [2002] fanden massive Unterschiede im «Craving» von Alkoholkranken und pathologischen Glücksspielern und sahen erhebliche Probleme, bei Glücksspielern zwischen Craving- und Depressionsratings zu unterscheiden.
Die damalige Konzeptalternative war unser Neurosemodell. Moran [1970] hatte in England die erste Studie zur Differenzierung von Subtypen pathologischer Glücksspieler publiziert und am häufigsten solche mit einer neurotischen Primärstörung gefunden. Nach dem Neurosemodell [vgl. Hand, 1992, 1997, 1998b] erlebt ein Mensch in negativer emotionaler Befindlichkeit (bei gleichzeitiger erlernter Hilflosigkeit) durch Zufallskontakte mit Glücksspielsituationen (möglichst auch mit einigen Gewinnen) Ablenkung bzw. eine Reduktion seiner negativen Befindlichkeit. Hat er keine besseren Alternativen zur Vermeidung negativer Befindlichkeiten, so ist er gefährdet, pathologischer Glücksspieler zu werden. Die Entzugssymptome des Suchtmodells werden im Neurosemodell als typische Angst-Depressions-Symptome interpretiert, die gespürt werden, sobald das Vermeidungsverhalten, das Glücksspielen, nicht mehr möglich ist. Später kommt ein zusätzlicher Teufelskreis negativer Verstärkung hinzu: es muss auch weitergespielt werden, um die verlustinduzierte zusätzliche negative Befindlichkeit durch Gewinne zu reduzieren (chasing) [Lesieur, 1984].

Im Rahmen der jahrzehntelangen Diskussion, ob Süchte Zwänge oder Zwänge Süchte sind, war dieses Modell des Glücksspielens damals eigentlich ein «Zwangsneurosenmodell».

Die in den 80er Jahren bestehende Unklarheit bezüglich der Zuordnung pathologischen Glücksspielens zu Zwang oder Sucht wurde besonders deutlich am Beispiel der Darstellung «führender Merkmale einer Abhängigkeit» von Schumacher [1981], der eine Sucht dann diagnostizierte, wenn Symptome «nach Art einer Zwangsstörung» vorlagen [s. auch Hand, 1992].

Implizite und explizite Modelle negativer Verstärkung pathologischen Glücksspielens in der internationalen Literatur

Etliche Autoren haben zwischen ca. 1965 und 1995 negative Verstärkung bereits implizit oder explizit als wesentliche Funktion pathologischen Glücksspielens aufgeführt [Bräutigam, 1958; Tyndel, 1963; Moran, 1970; Hand und Kaunisto, 1984; Glen, 1985; Zimmermann et al., 1985; Taber et al., 1987; McCormick et al., 1987, 1989; Ottinger, 1988; Coreless und Dickerson, 1989; Blaszczynski et al., 1990; Dickerson et al., 1991; Gazzaniga, 1992; Hand, 1992; Baumeister et al., 1994; Lumley und Roby, 1995; Coman et al., 1997; Daughters et al., 2002; Wildman II, 1997; Literaturzusammenfassung in O'Conner et al., 2004]. Beispielhaft seien die folgenden Referenzen angeführt:

- Impulskontrollstörungen, einschließlich Drogensucht, als erfolgloser Copingversuch mit Schuldgefühlen/Depression/Angst [Tyndel, 1963], psychischem Schmerz und negativen Lebenserfahrungen [Zimmermann et al., 1985; Khantzian, 2002].

- Problematisches Glücksspielen als maladaptiver Versuch, mit unterschiedlichen unangenehmen Gefühlszuständen - 


\begin{tabular}{|c|c|c|}
\hline $\begin{array}{l}\text { SOZIALES } \\
\text { GLÜCKSSPIELEN }\end{array}$ & $\begin{array}{l}\text { PATHOLOGISCHES } \\
\text { GLÜCKSSPIELEN }\end{array}$ & $\begin{array}{l}\text { «SÜCHTIGES»(?) } \\
\text { GLÜCKSSPIELEN }\end{array}$ \\
\hline $\begin{array}{l}\text { C+-Modell: }^{+} \\
\text {Positive Verstärkung } \\
\end{array}$ & $\begin{array}{l}\text { \&--Modell: } \\
\text { Negative Verstärkung }\end{array}$ & $\begin{array}{cl}\text { Präsuizidales } \rightarrow & \begin{array}{l}\text { Parasuizidales } \\
\text { Verhalten }\end{array} \\
\end{array}$ \\
\hline $\begin{array}{l}\text { SPASS AM SPIEL } \\
\text { (Action Orientierung) }\end{array}$ & $\begin{array}{l}\text { ESCAP(E)-ISM } \\
\text { (aktive Meidung) } \\
\text { • Alltagsleben }=\ll \text { Schmerz» } \\
\text { (Depression, Angst, Schuldgefühle, } \\
\text { Ambivalenz) }\end{array}$ & $\begin{array}{l}\text { NICHTGEWUSSTE INTENTION ZUM } \\
\text { SUIZID } \\
\text { (passive Meidung) }\end{array}$ \\
\hline $\begin{array}{l}\text { STIMULATION } \\
\text { bei Langeweile } \\
\text { NOW - ISM } \\
\text { Instant Befriedigung } \\
\text { (geweckter) Bedürfnisse }\end{array}$ & $\begin{array}{l}\text { - Intrapsychische Funktionalität: } \\
\text { - Meidung von Schmerz/negativer } \\
\text { Befindlichkeit im ziellosen Glücks- } \\
\text { spiel; Schein-/ Märchenwelt der } \\
\text { Glücksspielsituation) } \\
\text { - Vermeidung von Selbstwertverlust und } \\
\text { «Schande» durch Spielverlust (chasing) }\end{array}$ & $\begin{array}{l}\text { •Intrapsychische Funktionalität: } \\
\text { - Abreaktion selbstzerstörerischer } \\
\text { Impulse } \\
\text { - Durch Verluste Erhöhung des } \\
\text { «inneren Drucks» zur Suizid- } \\
\text { handlung }\end{array}$ \\
\hline & $\begin{array}{l}\text { - Interaktionelle Funktionalität: } \\
\text { - Abreaktion von Aggressionen } \\
\text { gegen enge Beziehungsperson } \\
\text { - Provokation der engen Beziehungs- } \\
\text { person (z. B. zur Trennung) }\end{array}$ & $\begin{array}{l}\text { - Interaktionelle Funktionalität: } \\
\text { - z. B. Rache am Partner (Vernich- } \\
\text { tung auch von dessen Vermögen) }\end{array}$ \\
\hline MATERIELLE SINNORIENTIERUNG & MANGEL AN SINNORIENTIERUNG & VERLUST DER SINNORIENTIERUNG \\
\hline
\end{tabular}

Abb. 1. Motivation zum Glückspielen.

vor allem Depression oder Langeweile - zurechtzukommen [Blaszczynski, et al., 1990; Baumeister et al., 1994].

- Stress und Angst als Risikofaktoren für pathologisches Glücksspielen [Coman et al., 1997]. Mangelnde Toleranz für negativen Affekt und Distress als hohes Rückfallrisiko [Daughters et al., 2002].

- Unerträgliche Minderwertigkeitsgefühle als Ursache chronischer negativer Lebenseinstellung und hoffnungsloser Depression mit dem Versuch, übermäßig zu kompensieren, z.B. durch Arbeiten, Glücksspielen etc. [Ottinger, 1988].

- Gehäufte negative, traumatische Lebensereignisse führen zu Schuldgefühlen, ähnlich wie bei der posttraumatischen Belastungsstörung, die auch bei 30\% der pathologischen Glücksspieler gefunden wurde; dies führt zu «erlernter Hilflosigkeit» [McCormick et al.; 1987, 1989; Niederland, 1967, 1984; Taber et al., 1987; Jacobs, 1989, 2003].

- Frühe Hypothesen zur Trennung von zwei Subgruppen pathologischer Glücksspieler - mit positiver oder negativer Verstärkung (in heutiger Terminologie) - finden wir schon bei Bräutigam [1958], Custer und Milt [1985], Taber et al. [1987] und Lesieur [1988].

Diese Modellvorstellungen in den 80er und 90er Jahren waren erheblich durch die Klientel mitbestimmt, die in den jeweiligen Therapieeinrichtungen im Vordergrund stand, z.B. Personen mit stoffgebundenen Süchten und problematischem/pathologischem Glücksspielen in den stationären Einrichtungen der Veteran Administrations (VA) Hospitals in den USA einerseits und die Vermeidungsspieler in der Hamburger Verhaltenstherapie-Ambulanz andererseits [s. auch Blanco et al., 2001].

\section{Therapeutische Konsequenzen aus dem Sucht- und dem (Zwangs-)Neurosenmodell}

Die verschiedenen theoretischen Konzepte hatten sehr unterschiedliche therapeutische Konsequenzen. Die wesentlichen Merkmale und Inhalte der damaligen Suchttherapie nach dem Modell der Anonymen Alkoholiker (AA) bzw. der Anonymen Spieler (GA) in den VA Hospitals (zu den heutigen, wesentlich komplexeren Behandlungsstrategien in deutschen Spielsucht-Kliniken s. dagegen z.B. Petry [2003]) waren Folgende:

Die initial stationäre «Entzugsbehandlung» und die ambulante Weiterbetreuung - in erster Linie in Selbsthilfegruppen waren identisch mit der abstinenzorientierten Behandlung bei stoffgebundenen Süchten. Nicht selten wurde sogar vor Aufnahme in die Therapie das Erreichen eines Abstinenzverhaltens gefordert. Stationär standen Isolation und sensorische Deprivation von der bevorzugten Aktivität (Glücksspielen) im Mittelpunkt; Isolation von der sozialen Realität (einschließlich der Familie); kontinuierliche Konfrontation mit dem «Ich-bin-süchtig»-Modell (erzwungener Glaube); Entzug von Radio, Fernsehen und Presse (da diese Trigger für Glücksspielverhalten enthalten) und Einförmigkeit des sozialen Umfeldes (alle Mitpatienten sind süchtig, viele Therapeuten ebenfalls) mit Uniformität des Krankheitsmodells. In der ambulanten Stabilisierungsphase konstante Belohnung für das «Ich-bin-süchtig»-Selbstbild («von der Scham zum Stolz»); ständige Beschäftigung mit «Impulskontrolle» als Süchtiger und oft hohe externe Kontrolle (z.B. durch Angehörige oder Selbsthilfegruppe) als Gegengewicht zum eigenen Suchtrisiko. Die Selbsthilfegruppen (für den Patienten GA; für die An- 
Tab. 1. «Sucht-Abstinenz-Verhalten»- ein sinnvolles Ziel bei «nichtstoffgebundenen Abhängigkeiten»?*

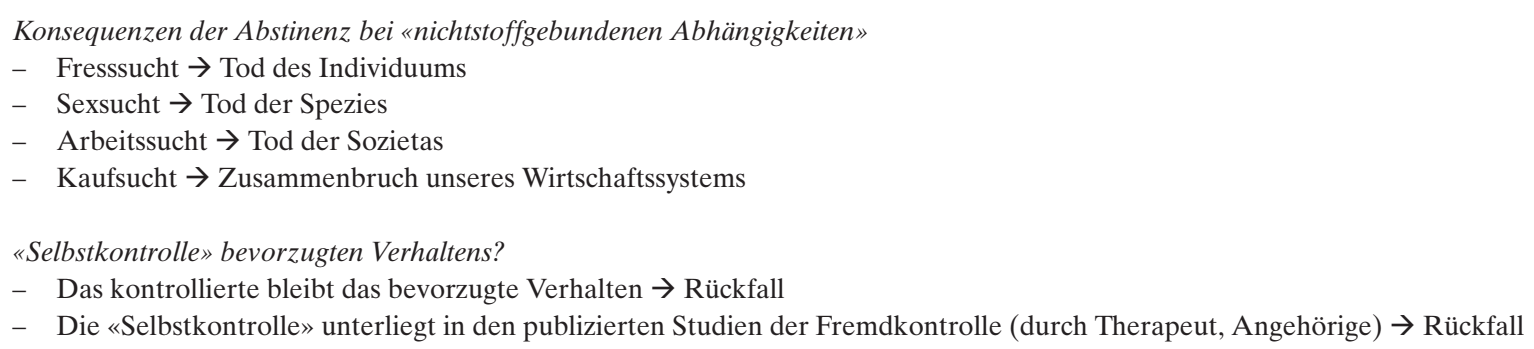

Konsequenzen der «Fremdkontrolle» bevorzugten Verhaltens

- «Freiwillig» (über Schuldgefühle) akzeptierte Fremdkontrolle $\rightarrow$ Rückfall

- Druck erzeugt Gegendruck - je gesünder das Individuum, umso wirksamer Brehms «Reaktanz»-Modell $\rightarrow$ Rückfall

- Externe Kontrolle eines Verhaltens (z.B. Glücksspielen) bedingt externe Kontrolle weiterer Verhalten («Wo warst Du wann?») $\rightarrow$ Reaktanz $\rightarrow$ Rückfall

Alternativen

- Aufbau und Förderung von attraktiverem «Alternativverhalten»: «Competing response»; neues Verhalten ersetzt altes Verhalten sowohl bezüglich negativer wie positiver Verstärkung. Hier wird ein «Rückfall» zum Signal für neue (alte) Fehler in der Lebensführung

*Cave: «Abhängiges» Verhalten = subjektiv bevorzugtes Verhalten.

gehörigen GAMANON; für Jugendliche in der Familie TEENANON) haben in der Regel die entscheidende Bedeutung; Einzelkontakte für Patient und PartnerIn beim Suchttherapeuten können hinzukommen.

Selbsthilfegruppen für Glücksspieler können zwar wertvolle Unterstützung geben, scheinen aber sehr viel weniger Erfolg im Hinblick auf Abstinenz zu erzielen als die Anonymen Alkoholiker [z.B. Brown, 1987]. Aus systemischer Sicht ergibt sich dabei folgende Hypothese: Bei sehr lebens- und konfliktängstlichen Personen, bei denen «süchtiges» Spielen eine Flucht aus dem Erleben dieser Ängste darstellte, vermag vermutlich die Etablierung einer zwanghaften Gegensteuerung und Kontrolle [vgl. Matussek, 1958] und die Einbindung in ein quasi religiöses Hilfesystem eine alternative Bewältigungsmöglichkeit für die allgegenwärtige Angst darstellen. Darüber hinaus könnte die zeitweise Trennung der Familienmitglieder durch Besuch ihrer jeweils spezifischen Selbsthilfegruppe Konflikte im Familiensystem und dadurch die Rückfallrisiken reduzieren (vgl. das Konzept der «Expressed Emotion» in der Schizophrenieforschung; z.B. Goldstein et al. [1986]). Es läge dann eine negative Verstärkung des Selbsthilfegruppenbesuches (als Ersatz für den Besuch der Glücksspieleinrichtung) durch passagere (familiäre) Spannungsreduktion vor.

Aus verhaltensanalytisch-systemischer Sicht haben wir bereits in der zweiten Hälfte der 80er Jahre das Abstinenzgebot bei nichtstoffgebundenen Abhängigkeiten für problematisch gehalten. Einige Argumente gegen das Abstinenzgebot sind in Tabelle 1 aufgeführt. Die Kritik basiert auf motivationspsychologischen Überlegungen zu den intrapsychischen und interaktionellen Funktionalitäten von Problemverhalten. Verhaltensexzesse sind im Augenblick des Handelns auf Grund der sofortigen negativen Verstärkung das subjektiv bevorzugte Verhalten [vgl. Hand, 1981, 2002a]. Werden die Funktiona- litäten des Problemverhaltens in der Therapie übergangen, gibt es massive Motivationsprobleme. Wir haben deshalb schon seinerzeit Kritik an ausschließlich symptomzentrierten verhaltenstherapeutischen Verfahren wie Aversionstherapie, Desensibilisierung und insbesondere «Selbstkontrolle» geübt. Diese Kritik galt auch für die damaligen «Selbstkontroll»Therapien, da sie nur unter Fremdkontrolle umgesetzt wurden (der Patient musste sich z.B. bei Partnerin abmelden, wenn er für eine festgelegte Zeit mit einem festgelegten Geldbetrag in eine vorher festgelegte Spielhalle zum Glücksspielen ging). Solange anstelle des subjektiv bevorzugten Problemverhaltens kein anderes subjektiv bevorzugtes Verhalten tritt, besteht permanent eine hohe Rückfallgefahr [vgl. Hand, 1981]. Ferner erzeugt jede Form von Druck in psychologisch noch relativ gesunden Individuen Gegendruck (Brehms Reaktanzprinzip) - ein weiterer rückfallfördernder Faktor. Schließlich bedingt die externe Kontrolle einer Verhaltensweise (z.B. Glücksspielen) die Kontrolle weiterer Verhaltensweisen (z.B. anderer aushäusiger Aktivitäten) im Alltagsleben.

Aus den in Tabelle 1 dargestellten Überlegungen haben wir seinerzeit ein therapeutisches Vorgehen abgeleitet [Klepsch et al., 1989b]. Es entsprach unserem multimodalen, auch kausal orientierten Vorgehen bei komplexer gestörten Patienten, insbesondere bei Zwangskranken [Hand, 2000, 2002b] - mit Ausnahme des gravierenden Unterschiedes, dass wir bisher bei pathologischen Glücksspielern keine symptomzentrierten Interventionen durchgeführt haben, während diese bei Zwangsstörungen entscheidend sind. Neben der Reduktion der ursächlichen Störungen sollen bessere Copingstrategien für den Umgang mit negativen Befindlichkeiten vermittelt und neue Erlebnisquellen für positive Verstärkung eröffnet werden. Damit sollte negative Verstärkung durch das Problemverhalten verzichtbar werden. Auf diese Weise müsste 
der (Pseudo-)«Suchtdruck» verschwinden und Kontakte mit Glücksspielsituationen dürften keine risikoreichen Reaktionen mehr auslösen. Dieses schien sich in drei Langzeitkatamnesen in Form von Anwendungsbeobachtungsstudien für zwei Drittel der Therapie- und Katamneseteilnehmer - unabhängig von der jeweils notwendigen Therapiedauer - zu bestätigen [Klepsch et al., 1987, 1989b; Wlazlo et al., 1987]. Zum Beweis dieser Hypothese sind jedoch weitere, insbesondere externe Studien erforderlich.

Schon über zwei Jahrzehnte vorher entwickelten tiefenpsychologisch-analytisch orientierte Autoren ein ähnliches Grundkonzept mit ähnlichen Therapiezielen, aber einem völlig anderen therapeutischen Vorgehen. Matussek [1958] postulierte, dass eine süchtige Fehlhaltung nicht beseitigt wäre, solange sie noch durch zwanghafte Mechanismen zurückgehalten werden müsse, z.B. durch «zwanghaft-rituelle Befolgung von Vorschriften»; der Süchtige sei im psychotherapeutischen Sinne erst dann geheilt, wenn die süchtige Tendenz beseitigt bzw. abgeschwächt sei. Dies heißt in der Konsequenz: Nur wer die Psychotherapie nicht akzeptieren oder nutzen kann, möge sich im zwanghaften Ritual vor einem Rückfall in «Suchtverhalten» schützen.

Demgegenüber hatte der Analytiker Wurmser [zit. n. Vent, 1999] schon früh die Süchte den schweren Neurosen zugeordnet und als Wiederholungszwang interpretiert, der vor allem der Konfliktbewältigung und Affektregulierung diene (neuere Modelle zu «Sucht gleich Zwang» bei Dodes [1996] und Khantzian [2002]).

Unser eigenes Modell wurde aus klinischer Erfahrung und der Strategie multimodaler Verhaltenstherapie abgeleitet, noch ohne Kenntnisse der oben angeführten Modelle.

\section{Aktuelle therapeutische Modelle negativer Verstärkung pathologischen Glücksspielens}

$\mathrm{Zu}$ den aktuell in den USA am meisten diskutierten impliziten Modellen negativer Verstärkung pathologischen Glücksspielens - die ursprünglich für stoffgebundene Süchte entwickelt worden waren - zählen das ätiologische Modell der «frühen Traumatisierung mit dissoziativen Phänomenen» [z.B. Jacobs, 1989] und das funktionale Modell der «Selbstmedikation» [z.B. Khantzian, 2002]. Beide entstanden in ersten Ansätzen bereits Mitte der 80er Jahre, sind aber erst in den letzten Jahren auf den internationalen Glücksspielkongressen in den Vordergrund getreten.

\section{Das Modell der frühen Traumatisierung mit Dissoziation}

Nach dem «General Model of Addictions» [Jacobs, 1987, 1989, 2003] für stoffgebundene und nichtstoffgebundene Süchte sind «süchtige» Verhaltensweisen symptomatisch für folgende ursächliche Faktoren: Erhöhter Arousal; traumatische Kindheitserlebnisse; lang anhaltende Depression; begrenzte interaktionelle Copingfertigkeiten. Typische Merkma- le sind: unscharfe Realitätsprüfung; reduzierte selbstkritische Einstellung und dysphorische Stimmung; Induktion eines veränderten Bewusstseinszustandes. Auf diese Weise erleichtern süchtig machende Substanzen oder Verhaltensweisen wunscherfüllende - im Sinne eines Selbstheilungsversuches Phantasien mit einem veränderten Selbstbild oder auch die vollständige Flucht vor früheren oder aktuellen Stressoren. Sie ermöglichen so eine «vorübergehende Amnesie». Jacobs gibt an, dass bis zu $80 \%$ der pathologischen Glücksspieler Kindheitstraumata erlebt und davon wiederum etwa $80 \%$ während des Traumas dissoziiert hätten; dies erkläre die häufigen dissoziativen Zustände während des pathologischen Glücksspielens. Primär abstinenzorientierte Therapien stünden im Widerspruch zu den initialen Zielen der Süchtigen. Die Therapie müsse vielmehr folgende Schwerpunkte haben: 1. Stress-Management-Training; 2. psychodynamische und kognitiv-verhaltenstherapeutische Interventionen zum Abbau der Nachwirkungen der frühen Traumata; 3. Training von Copingfertigkeiten; 4. Beratung bzw. Psychoedukation der wichtigsten Bezugspersonen. Die Bearbeitung falscher Glücksspielüberzeugungen hat in diesem Modell eher geringe Bedeutung.

Außerhalb der Expertenszene für pathologisches Glücksspielen in den USA scheint Jacobs' Modell unter Suchtexperten in den USA aber wenig bekannt zu sein (vgl. das Interview in diesem Heft). Es steht auch im Widerspruch zu der heute überwiegenden Meinung, dass pathologische Glücksspieler eine ätiologisch sehr heterogene Gruppe darstellen. Als Modell für eine Untergruppe pathologischer Spieler erscheint es aber therapeutisch nützlich [vgl. McCormick et al., 1987, 1989].

\section{Das Modell der Selbstmedikation}

Ein in den USA wesentlich breiter akzeptiertes Modell negativer Verstärkung bei Süchten ist das der «SelbstmedikationsHypothese», das von Khantzian zuerst vor allem für Heroinund Kokainsucht publiziert wurde [1985], dann modifiziert und auf alle «substance use disorders» erweitert [1997] und schließlich auch auf «Verhaltensstörungen» übertragen wurde [2002]. Dieses vielschichtige, tiefenpsychologisch orientierte Modell bezieht sich auch auf «aktuelle analytische Konstrukte von Süchten als Zwangsstörungen» [Khantzian, 2002], insbesondere auf Dodes [1996]. Damit geht also die Diskussion «Zwang, eine Sucht?» oder «Sucht, ein Zwang?» weiter. Kernhypothese des Selbstmedikationsmodells ist, dass Süchte und ähnliche Verhaltensweisen dazu dienen, Dysphorie oder unerträgliche affektive Befindlichkeiten zu reduzieren oder abzuwenden [Khantzian, 2002]. Bezüglich der Ursachen für Dysphorie und negative affektive Befindlichkeiten verweist Shaffer [2002, 2003] ausdrücklich darauf, dass die hohe psychiatrische Komorbidität von pathologischen Glücksspielern oft schon vor Auftreten der Sucht vorgelegen habe. Eine der wichtigsten Fragen an Betroffene sei: «What did the drug do for you when you took it first?». 
Tab. 2. Motivation zum Glücksspielen in zwei Studien*

\begin{tabular}{|c|c|c|}
\hline WAGER-Studie, USA [1988] & \multicolumn{2}{|c|}{ Nennungen, \% } \\
\hline \multicolumn{3}{|l|}{ Positive Verstärkung } \\
\hline Erwartung von Geldgewinn & \multicolumn{2}{|l|}{50,5} \\
\hline Zur Unterhaltung & \multicolumn{2}{|l|}{33,4} \\
\hline Um Spaß zu haben & \multicolumn{2}{|l|}{18,4} \\
\hline Aus Neugierde & \multicolumn{2}{|l|}{10,6} \\
\hline Aus Neigung & \multicolumn{2}{|l|}{4,1} \\
\hline Als Hobby & \multicolumn{2}{|l|}{3,6} \\
\hline \multicolumn{3}{|l|}{ Negative Verstärkung } \\
\hline Zur Ablenkung & \multicolumn{2}{|l|}{41,1} \\
\hline \multirow[t]{2}{*}{ ARI-Studie, Australien [1966] } & \multicolumn{2}{|c|}{ Nennungen, \% } \\
\hline & Männer & Frauen \\
\hline \multicolumn{3}{|l|}{ Positive Verstärkung } \\
\hline Erwartung von Geldgewinn & 42,0 & 21,7 \\
\hline Zur Unterhaltung & 29,5 & 0,0 \\
\hline \multirow[t]{2}{*}{ Zum Vergnügen } & 22,6 & 0,0 \\
\hline & $(94,1)$ & $(21,7)$ \\
\hline \multicolumn{3}{|l|}{ Negative Verstärkung } \\
\hline Zum Abbau von Stress & 18,4 & 24,6 \\
\hline Zum Abbau von Langeweile & 0,0 & 36,5 \\
\hline Zur Vermeidung von Einsamkeit & 0,0 & 31,3 \\
\hline \multirow[t]{2}{*}{ Zum Abbau von Angst } & 0,0 & 12,2 \\
\hline & $(18,4)$ & $(104,6)$ \\
\hline
\end{tabular}

*In beiden Studien Mehrfachnennungen möglich.

\section{Motivations-Modifikationsmodelle}

Neben diesen beiden Therapiemodellen sind motivationspsychologische Ansätze in der «Sucht»-Behandlung in den Vordergrund getreten. Kennzeichnend für diese ist die Abwendung von der üblichen «Action»-Orientierung der Therapie (z.B. umgehend Abstinenz durchsetzen, Besuch von Selbsthilfegruppen einleiten usw.) hin zu einem «Stadienmanagement» (z.B. Klärung der Veränderungsmotivation; woher kommt Leidensdruck?; was will der Betroffene wirklich?). Vor allem das «Motivational Interviewing» [Miller und Rollnick, 1991] und das «Stage-of-Change»Modell mit dem «Stage-Management-Treatment» [Prochaska et al., 1994, 1999] sind als niederschwellige Ansätze zur Einleitung von Veränderungen breit akzeptiert. Damit sollen mehr als die bisher nur geschätzten 4-20\% [Petry, 2002, bzw. Prochaska, 2002] der Personen mit Suchtproblematik für eine Hilfesuche motiviert und die Therapeuten aus ihrer «demoralized helplessness» herausgeführt werden. Personen mit chronifiziertem, risikoreichem Problemverhalten sollen dann - sozusagen mit einer erweiterten «KanferRegel»-zu Verhaltensveränderungen motiviert werden. Kanfers Kernsatz, «Join the client where he is ...», wird also erweitert um «... and lead him where he doesn't want to go» [Costikyan, 2002].
Die therapeutischen Konsequenzen aus diesen Ansätzen gehen also in erster Linie in Richtung Psychotherapie. Das drückte sich auch im Titel der Las-Vegas-Konferenz 2002 der Harvard Medical School's Division on Addictions aus: «ReThinking Addiction - How Gambling and Other Behavioral Addictions are Changing the Concept and Treatment of Alcohol and Substance Use Disorders». Während in den 80er Jahren massiver Druck ausgeübt wurde, den verhaltensanalytisch-motivationalen Ansatz zu Gunsten einer an AA und GA orientierten Suchttherapie aufzugeben, geht die Tendenz jetzt teilweise in die entgegengesetzte Richtung - wobei häufig von Sucht gesprochen und Zwang oder Neurose gemeint wird! Es lässt sich allerdings noch nicht absehen, ob und wann die immer noch kontroverse Diskussion zu einem Konsens führen wird.

\section{Erste Daten zu positiver und negativer Verstärkung beim (pathologischen) Glücksspielen}

Zu motivationalen Aspekten des Glücksspielens gibt es kaum epidemiologische Studien. 1993 wurden in den USA bundesweit Telefoninterviews mit 1522 zufällig ausgewählten, erwachsenen US-Bürgern durchgeführt. Von diesen hatten 937 $(61,6 \%)$ in den 12 Monaten vor dem Interview an Glücksspielen teilgenommen (Intensität nicht angegeben). Ausgehend davon, dass damals $1,14 \%$ der erwachsenen amerikanischen Bevölkerung pathologische Glücksspieler [Shaffer und Hall, 2001] und weitere 3,8\% Problemspieler [Petry, 2002b] waren, dürften von den 1522 befragten Personen 17 pathologische und weitere 58 Problemspieler gewesen sein. Die Ergebnisse dürften also zu 95\% an sozialen Glücksspielern gewonnen sein. In Tabelle 2 wird diese Studie als WAGER-Studie angeführt, da die Daten dort publiziert sind [The Wager, 1998]. In Australien führte das Addiction Research Institute (ARI) in Melborne 1996 Telefoninterviews mit 720 Anrufern ihres telefonischen Beratungsdienstes durch [Coman et al., 1997; The Wager, 2000]. Davon erwiesen sich 234 als Problemspieler. Trotz der erwähnten diagnostischen Unschärfen in beiden Studien kann angenommen werden, dass in ersterer überwiegend soziale Spieler und in letzterer überwiegend pathologische und Problemspieler erfasst wurden. Die Ergebnisse beider Studien lassen sich vergleichend - unter positiver und negativer Verstärkung als Hauptmotivation zum Glücksspielen zusammenfassen (s. Tab. 2).

Diese Daten lassen natürlich nur sehr vorsichtige Schlussfolgerungen zu. Trendmäßig lässt sich jedoch feststellen, dass nicht überraschend - bei den sozialen Spielern positive Verstärkung die entscheidende Rolle spielt. Dies ist auch in Deutschland bei etwa $70 \%$ der sozialen Spieler so [Hand, 1986].

Überraschend ist dagegen das extreme Überwiegen positiver Verstärkung bei den männlichen Problemspielern und das nahezu umgekehrte Ergebnis bei weiblichen Problemspie- 


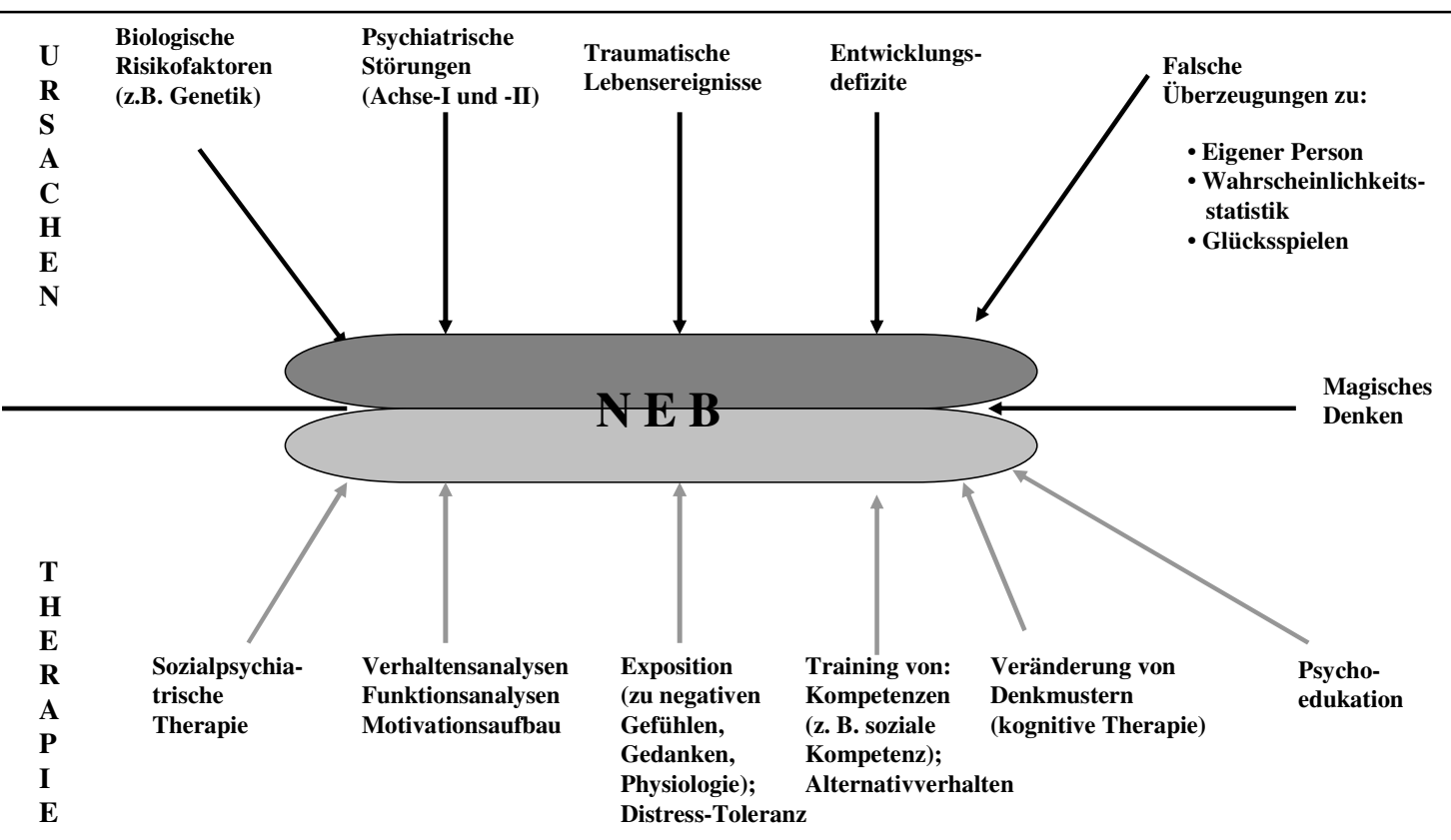

Abb. 2. Negative Befindlichkeit (NEB) und pathologisches Glücksspielen.

\section{MULTIMODALE (VERHALTENS-)THERAPIE}

(MEDIKAMENTÖSE THERAPIE ?)

lern in Australien. In den USA bestand schon vor 20 Jahren der Eindruck, dass weibliche pathologische Glücksspieler im Gegensatz zu männlichen eher mit negativer Verstärkung spielten [Lesieur, 1988]. Als mögliche Erklärung wird angeführt, dass in den USA, Australien und Neuseeland das Glücksspielen traditionell eine Domäne der Männer ist, die aufgrund der historischen Entwicklung positiv risikofreudig dem Glücksspiel gegenüber orientiert seien, demgegenüber seien die weiblichen (Vermeidungs-)Spieler weit überwiegend in der geschichtlichen Entwicklung des Glücksspielens «Späteinsteiger».

Kürzlich wurden 2 Untersuchungen an pathologischen Glücksspielern in Therapieeinrichtungen in Brasilien [Tavares et al., 2001] und Spanien [Ibáñez-Cuadrado et al., 2001] vorgestellt, die ein sehr hohes Überwiegen negativer Verstärkung bei den weiblichen (88\%) und immerhin noch ein fast ausgewogenes Verhältnis von positiver und negativer Verstärkung bei den männlichen Glücksspielern fanden; Glücksspielen wurde dabei «als Möglichkeit, Problemen auszuweichen und schlechte Gefühle zu vermeiden» angegeben. Bezüglich der Glücksspielerinnen sind die Ergebnisse denen der oben angeführten Studien sehr ähnlich. Bezüglich der männlichen Vermeidungsspieler weichen sie aber deutlich nach oben ab. Es deuten sich hier also erhebliche kultur- und geschlechtsspezifische Unterschiede an.

Die Informationen in den Studien aus Brasilien und Spanien wurden mit Hilfe selbst erstellter strukturierter Interviews in Anlehnung an DSM-IV erhoben, die Ergebnisse der englischsprachigen Studien mit Hilfe einfacher Fragebögen. Interna- tional existiert bisher kein valides und reliables Selbst- oder Fremdrating-Instrument. Der Kurzfragebogen zum Glücksspielverhalten, KFG, von Petry [2003] erlaubt im deutschen Sprachraum eine etwas detailliertere Erfassung der Funktionalität des (pathologischen) Glücksspielens. Das für diese Fragestellung spezifisch entwickelte Hamburger-Glücksspiel-Inventar, HGI, [Hand et al., persönliche Daten] befindet sich gegenwärtig in einer ersten Evaluation [Cordes, 2004]. In der interdisziplinären Suchtforschungsgruppe Berlin (ISFB) wird ein Fragebogen zur «differenzierten Anamnese von belohnenden Verhaltensweisen» (FDAV) evaluiert [Grüsser-Sinopoli, 2003]. Die Ergebnisse der bisher publizierten Studien sind also bei weitem noch nicht abgesichert. Sie «passen» aber sehr schön in klinische Modelle - worin allerdings auch die Gefahr vorschneller, unkritischer Akzeptanz besteht.

\section{Ein aktuelles verhaltenstherapeutisches "Neurosenmodell» pathologischen Glücksspielens}

Von der Grundorientierung her unterscheidet sich das verhaltenstherapeutische Modell unserer Arbeitsgruppe kaum von den früheren tiefenpsychologisch-analytischen Konzeptbildungen oder dem stark psychodynamisch geprägten Modell der Selbstmedikation und den oben angeführten motivationalen Interventionen. Tiefgehend unterscheidet sich unser Vorgehen dagegen von den früheren verhaltenstherapeutischen symptomgerichteten Interventionen [Überblicke in Klepsch et al., 1989b; Blaszczynski und Silove, 1995; Meyer und Bach- 
Tab. 3. Glücksspieler-Subtypen und Verhaltenstherapie-Indikationen

\begin{tabular}{|c|c|}
\hline \multicolumn{2}{|l|}{ Indizierte Verhaltenstherapie-Verfahren } \\
\hline $\begin{array}{l}\text { Action-Sucher [Lesieur, 1988] } \\
\text { C+(positive Verstärkung)-Glücksspieler [Hand, 1992, 1998b] }\end{array}$ & $\begin{array}{l}\text { Escape-Sucher [Custer und Milt, 1985; Lesieur, 1988] } \\
\text { C }^{-} \text {(negative Verstärkung)-Glücksspieler [Hand, 1992, 1998b] } \\
\text { Selbstmedikation-Glücksspieler [Khantzian, 2002] }\end{array}$ \\
\hline Symptom-Verfahren & «Ursachen»-Verfahren \\
\hline $\begin{array}{l}\text { Psychoedukation über } \\
\text { - Zufallsstatistik } \\
\text { - } \quad \text { «Psycho-Fallen» in den verschiedenen Glücksspielangeboten }\end{array}$ & Sehr sorgfältige psychopathologische Differentialdiagnostik \\
\hline $\begin{array}{l}\text { Klärung und Veränderung der persönlichen Fehlannahmen über } \\
\text { - Glücksspiele } \\
\text { - } \text { eigene Person }\end{array}$ & $\begin{array}{l}\text { Ausführliche biographische Analyse unter Einbeziehung } \\
\text { systemischer Aspekte }\end{array}$ \\
\hline $\begin{array}{l}\text { Klärung der Bedingungen für die Entwicklung vom sozialen zum } \\
\text { pathologischen Spieler }\end{array}$ & $\begin{array}{l}\text { Hierarchisierte, multimodale Hypothesenbildung und } \\
\text { Interventionen }\end{array}$ \\
\hline $\begin{array}{l}\text { Genusstraining (Aufbau alternativer positiver } \\
\text { Verstärkungserlebnisse) }\end{array}$ & $\begin{array}{l}\text { Tagesprotokolle der Patienten zu Ereignissen und Befinden vor, } \\
\text { während und nach dem aktuellen Spielen }\end{array}$ \\
\hline & Aufbau von Verhaltensaktiva, Abbau von Verhaltensdefiziten \\
\hline & Genusstraining (Aufbau positiver Verstärkungserlebnisse) \\
\hline «Ursachen»-Verfahren? & Symptom-Verfahren? \\
\hline
\end{tabular}

mann, 2000; Petry, 2003; O’Connor et al., 2004]. Auch für diese Therapien wurden aber - ebenso wie für neue kognitive, symptomzentrierte Ansätze [z.B. Ladouceur et al., 2001, 2003; Sylvain et al., 1997; Kidman, 2003] - teilweise beeindruckende Therapieerfolge beschrieben - wenngleich diese und unsere eigenen Katamneseergebnisse z.B. von Petry [2003] oder O'Connor et al. [2004] bezüglich ihrer Aussagekraft eher skeptisch beurteilt werden.

In den 90er Jahren haben wir ein Kontinuum-Modell der Motivation zum Glücksspielen vorgestellt [Hand, 1992, 1997], das sich auf dem Hintergrund des aktuellen Diskussionsstandes gut erweitern lässt (Abb. 1). Dieses Modell des Glücksspielens ging - wie unser Modell der unterschiedlichen Funktionen des Zwangsverhaltens [Hand, 2000, 2002b] - von einem Verhaltenskontinuum aus: vom sozialen Glücksspielen (das über positive Verstärkung stabilisiert wird), über das Vermeidungsspielen (das über negative Verstärkung stabilisiert wird), zum seinerzeit sog. süchtigen Glücksspielen (womit ein primär selbstdestruktives, rasch zum «sozialen Suizid» führendes Glücksspielverhalten gemeint war, mit der Hypothese, dass vor Aufnahme des exzessiven Glücksspielens aus anderen Gründen eine präsuizidale Grundverfassung vorlag). Für die Behandlung einer wesentlichen Untergruppe von pathologischen Glücksspielern - aber auch einer Untergruppe von Patienten mit Trichotillomanie und Kleptomanie [Neudecker und Rufer, 2004; Sauke, 2004] sowie vereinzelt bei «Kaufsucht» [Hand, 1998a] - hat sich die Definition der Vermeidungsspieler (s. Abb. 1) als ausgesprochen hilfreich erwiesen.
Die daraus sich ergebenden, multimodalen und individuell unterschiedlich zusammenzustellenden verhaltenstherapeutischen Interventionen lassen sich abstrahiert in einem Ursachen- und Therapiemodell bei Glücksspielern mit vor allem negativer Verstärkung und, jeweils in Teilbereichen spezifisch abgewandelt, wohl auch für andere Zwangsspektrumsstörungen darstellen (Abb. 2). In der oberen Hälfte der Abbildung finden wir typische, heterogene (mit-)ursächliche Faktoren, die zu dem relativ homogenen Problemverhalten führen können. In der unteren Hälfte sind die jeweils zugehörigen Interventionen aufgeführt, deren Bedeutung im Einzelfall von dem Vorliegen bzw. Überwiegen einzelner oder mehrerer Risikofaktoren abhängt. Für den einzelnen Patienten ist daraus jeweils das spezifische, hierarchisierte Störungs- und Behandlungsmodell abzuleiten [z.B. Hand, 2002a]. Aus der initialen Motivations- und Beziehungsanalyse ergibt sich der Zeitpunkt für die Einleitung verändernder Interventionen. Die Analyse der intrapsychischen (einschließlich der Verstärkermechanismen) und interaktionellen Funktionalitäten des Problemverhaltens bestimmt den Gesamtbehandlungsplan entscheidend mit. Die Behandlung kann daher, je nach Indikation, kurz und fokussiert in Einzel-, Paar- oder Familiensettings durchgeführt, oder aber als multimodale Intervention über längere Zeit erforderlich werden. Letztendlich handelt es sich also um eine zwar für pathologische Glücksspieler modifizierte, im Übrigen aber heute in Deutschland in der Patientenversorgung übliche, multimodale Verhaltenstherapie. 
Tab. 4. Glücksspieler-Subtypen und Pharmakotherapie-Indikationen nach Rosenthal [2004]

\begin{tabular}{|c|c|}
\hline \multicolumn{2}{|l|}{ Indizierte Pharmakotherapie-Verfahren } \\
\hline $\begin{array}{l}\text { Action-Sucher [Lesieur, 1988] } \\
\text { C+(positive Verstärkung)-Glücksspieler [Hand, 1992, 1998b] }\end{array}$ & $\begin{array}{l}\text { Escape-Sucher [Custer und Milt, 1985; Lesieur, 1988] } \\
\text { C}^{-} \text {(negative Verstärkung)-Glücksspieler [Hand, 1992, 1998b] } \\
\text { Selbstmedikation-Glücksspieler [Khantzian, 2002] }\end{array}$ \\
\hline $\begin{array}{l}\text { Opioid-Antagonisten } \\
\text { Naltrexon } \\
\text { Naltrexon + SSRI } \\
\text { Nalmefen } \\
\text { Cave: Iatrogene Dysphorie/Depression }\end{array}$ & $\begin{array}{l}\text { Trizyklische Antidepressiva } \\
\text { Imipramin }\end{array}$ \\
\hline $\begin{array}{l}\text { Beta-Blocker } \\
\text { Bisher keine Studie; Empfehlung von Rosenthal [2004] }\end{array}$ & $\begin{array}{l}\text { Selektive Serotonin-Wiederaufnahmehemmer (SSRI) } \\
\text { Fluvoxamin } \\
\text { Fluoxetin } \\
\text { Paroxetin } \\
\text { Citalopram }\end{array}$ \\
\hline Mood Stabilizer? & $\begin{array}{l}\text { Mood Stabilizer } \\
\text { Lithium } \\
\text { Carbamazepin } \\
\text { Valproinsäure }\end{array}$ \\
\hline Placebo & Placebo \\
\hline
\end{tabular}

Die verhaltenstherapeutische Forschungsliteratur berichtet dagegen überwiegend die erfolgreiche Behandlung pathologischer Glücksspieler mit symptomgerichteten, neuerdings insbesondere kognitiven, Interventionen in eher wenigen einzeloder gruppentherapeutischen Sitzungen. Daraus ergibt sich die Frage, ob unterschiedliche Patientengruppen mit dem für sie jeweils angemessenen Verfahren behandelt wurden oder ob zumindest kurzfristig jede Therapie erfolgreich ist, unabhängig von der durchgeführten Intervention. Für Letzteres könnte die sehr hohe Placebo-Erfolgsquote in den Pharmastudien sprechen [Petry, 2002a; Rosenthal, 2004].

Die Subgruppe mit (überwiegend) negativer Verstärkung ist heute durch eine Subgruppe mit (überwiegend) positiver und eine weitere mit sowohl positiver wie negativer Verstärkung pathologischen Glücksspielens in dem Modell (Abb. 1) zu ergänzen, mit den daraus hypothetisch abzuleitenden therapeutischen Konsequenzen für die Verhaltens- wie auch die Pharmakotherapie.

\section{Zusammenfassung der Hypothesen für Therapie und Forschung}

Grundsätzlich sind folgende (Verstärker-)Untergruppen pathologischer Glücksspieler denkbar:

1. Negative Verstärkung ohne frühere Erfahrungen mit sozialem Glücksspielen und dessen positiver Verstärkung (Vermeidungsspieler; escape-seeker).

2. Positive Verstärkung mit einer Vorgeschichte positiv verstärkten sozialen Glücksspielens (action-seeker).

3. Positiv verstärktes soziales Glücksspielen geht in primär positiv verstärktes pathologisches Glücksspielen über; sekundär entwickelt sich zusätzlich negativ verstärktes pathologisches Glücksspielen, über das die negativen Glücksspielfolgen (z.B. finanzielle Verluste) «ausgeblendet» werden - lustgesteuertes Zielverhalten wird auch frustgesteuertes Vermeidungsverhalten.

Pathologische Glücksspieler mit überwiegend positiver Verstärkung benötigen Psychoedukation und kognitive Interventionen zur Beseitigung ihrer Fehlannahmen über Glücksspielen und über die eigene Person. Die Indikation für weitere Interventionen ergibt sich aus den Verhaltensanalysen.

Vermeidungsspieler benötigen ursachenbezogene Interventionen und die Vermittlung von Copingstrategien für den Umgang mit negativen Befindlichkeiten. Die Indikation für zusätzliche kognitive und psychoedukative Interventionen zum Glücksspielverhalten selbst ergibt sich wiederum aus den Verhaltensanalysen.

Diese unterschiedlichen therapeutischen Konsequenzen sind in Tabelle 3 für die Verhaltenstherapie und in Tabelle 4 für die Pharmakotherapie [nach Rosenthal, 2004] dargestellt.

Pathologische Glücksspieler mit ausgeprägter negativer und positiver Verstärkung des Problemverhaltens benötigen von vornherein eine multimodale Therapieplanung sowohl hinsichtlich der ursachen- wie auch der symptomgerichteten Interventionen.

Die Bedeutung positiver und negativer Verstärkung bei sozialen, Problem- und pathologischen Glücksspielern scheint transkulturell und geschlechtsspezifisch sehr unterschiedlich zu sein. Zukünftige Therapiestudien und spätere Metaanalysen sollten durch Berücksichtigung dieser Unterschiede erheblich aussagekräftiger werden. 
Entsprechend den transkulturellen Unterschieden in der Verstärkerfunktion pathologischen Glücksspielens wurden in der kognitiven Symptomtherapie der Gruppe um Ladouceur möglicherweise vor allem männliche Glücksspieler mit vorwiegend positiver Verstärkung behandelt, während in unserer Arbeitsgruppe eher die Vermeidungsspieler Hilfe suchten. Hat jede Arbeitsgruppe intuitiv die für ihre Klientel zutreffende Behandlungsform gewählt [s. Blanco et al., 2001]?

Mit einer verlässlichen Testdiagnostik für die Verstärkerfunktion pathologischen Glücksspielens könnten Bildgebungsstudien zur Klärung der Frage beitragen, welche Untergruppe pathologischer Glücksspieler eher Patienten mit stoffgebundenen Süchten ähnelt und welche sich deutlich von diesen unterscheidet. Welche Bedeutung hat z.B. das «Belohnungssystem» für Vermeidungsspieler im Vergleich zu Action-Suchern? Welche Relevanz haben die kognitiven [z.B. Ladouceur et al., 2001, 2003] und die emotionalen [z.B. Milkman und Sunderwirth, 1998] Suchttheorien in diesem Kontext? Führt die aktuelle Forschung möglicherweise auch dazu, dass «Sucht» wieder, wie im 17. Jahrhundert, als «vollständige Hingabe an eine Tätigkeit» definiert wird [s. Shaffer, 1999, 2003; Kritik bei Holden, 2001]?

Von Custer und Milt [1985] bis heute haben viele Autoren postuliert, dass alles, was rasch, intensiv und verlässlich das Gefühl verändert, süchtig machen kann: «Where there is a reward, there is the risk of addiction» [Shaffer, 2002]. Aber nur $10 \%$ der Personen mit Drogenkontakt und nur etwa 3\% der Personen mit Glücksspielkontakt entwickeln ein entsprechendes pathologisches oder süchtiges Verhalten [Madras, 2002]. Welches sind die entscheidenden Variablen dafür, wenn es nicht das «Suchtmittel» ist? Auch zur Klärung dieser Frage könnte eine verbesserte Funktionsdiagnostik beitragen.

Eine die Funktionalität des Glücksspielverhaltens spezifischer berücksichtigende Therapie hätte vielleicht auch Chancen, den Anteil der Therapie aufsuchenden (und zu Ende führenden) pathologischen Glücksspieler über die bisher geschätzten 10\% [ Petry, 2002a] anzuheben.

Weder die bisher publizierten Verhaltenstherapie- noch die Pharmakotherapie-Studien haben die untersuchten Populationen nach dem Kriterium positiver und negativer Verstärkung des Problemverhaltens getrennt. Lassen sich anscheinend ähnliche Ergebnisse mit sehr unterschiedlichen Interventionen oder unterschiedliche Ergebnisse mit ähnlichen Interventionen darauf zurückführen, dass unterschiedliche Stichproben behandelt wurden? Die hier vorgetragenen Modelldarstellungen können vielleicht dazu beitragen, zukünftige Forschungen durch die Präzisierung spezifischer und relevanter Untergruppenbildungen zu verbessern. Bis dahin mögen sie Therapeuten Anregungen geben, klarere Hypothesen und Behandlungspläne zu erstellen und sich «ex juvantibus» einen «klinischen Eindruck» über die Nützlichkeit des Modells zu verschaffen. Bisherige Anwendungsbeobachtungen bei Patienten mit pathologischem Glücksspielen, Trichotillomanie und Kleptomanie lassen die Hoffnung berechtigt erscheinen. Die richtige Therapie für die pathologischen oder ProblemGlücksspieler sollte nicht länger gesucht werden. Eine «störungsspezifische» Therapie dürfte daher immer nur so gut sein, wie sie auch eine «funktionsspezifische», individuumbezogene Strategie beinhaltet.

\section{Literatur}

Baumeister RF, Heatherton TF, Tice DM: Losing Control - How and Why People Fail at Self-Regulation. San Diego, New York, Academic Press, 1994.

Blanco C, Moreyra P, Nunes E, Saiz-Ruiz J, Ibanez A: Pathological gambling - addiction or compulsion? Sem Clin Neuropsychiatry 2001;6:167-176.

Blaszczynski A, McConaghy N, Frankova A: Boredom proneness in pathological gambling. Psychol Rep 1990; 67:35-42.

Blaszczynski A, Silove D: Cognitive and behavior therapies for pathological gambling. J Gambling Stud 1995; 11: 195-220.

Bräutigam W: Psychotherapie bei Süchtigen. Nervenarzt 1958;29:445-451.

Brown RIF: Dropouts and continuers in Gamblers Anonymous. Part 4: Evaluation and summary. J Gambling Behav 1987;3:202-210.

Brister D, Brister P: The Vicious Circle Phenomenon Our Battle for Self-Control. Birmingham, Diadem Publishing, 1987.

Coman GJ, Burrows GD, Evans BJ: Stress and anxiety as factors in the onset of problem gambling: Implications for treatment. Stress Med 1997;13:235-244.

Cordes L: Eine erste Evaluation des neuen Hamburger Glücksspiel Inventars (HGI) - intrapsychische und interaktionelle Funktionalitäten des Glücksspielens. Diplomarbeit am FB Psychologie der Universität Hamburg, 2004.
Coreless T, Dickerson M: Gamblers' self-perceptions of the determinants of impaired control. Br J Addic 1989;84:1527-1537.

Costikyan N: Controversies in treating gambling and other addictive disorder. Konferenz «Rethinking Addiction», Las Vegas, 8-10.12.2002, abstr pp 7-8.

Custer RL, Milt H: When Luck Runs out: Help for Compulsive Gamblers and Their Families. New York, Facts on File Publication, 1985.

Daughters St. B, Lejuez CW, Strong DR, Breen RR, Lesieur HR: The relationship among negative affect, distress tolerance and latency to gambling treatment failure. Konferenz «Rethinking Addiction», Las Vegas, 8-10.12.2002, abstr p 22.

Davis JB: The Myth of Addiction, ed 2. Amsterdam, Harwood Academic Publishers, 1997.

Dickerson M, Cunningham R, England SL, Hincky J: On the determinants of persistent gambling III: Personality, prior mood, and poker-machine play. Int $\mathrm{J}$ Addict 1991;26:531-548.

Dodes LM: Compulsion and addiction. J Am Psychoanal Assoc 1996;44:815-835.

Gazzaniga MS: Nature's Mind: The Biological Routes of Thinking, Emotions, Sexuality, Language and Intelligence. New York, Basic Books, Harper Collins, 1992. Glen AM: Diagnosing the pathological gambler. J Gambling Behav 1985;1:17-22.
Goldstein MJ, Hand I, Hahlweg K (eds): Treatment of Schizophrenia - Family Assessment and Intervention. Berlin, Springer, 1986

Gross W: Sucht ohne Drogen. Frankfurt/M., Fischer Taschenbuch, 1990.

Grüsser-Sinopoli S: Interview. Dtsch Arztebl 2003;11: 514.

Hand I: Motivationsanalyse und Motivationsmodifikation im Erstkontakt; in Zuzan WD, Lacher R, Crombach-Seeber B (Hrsg): Erstkontakt. Wien, Literas-Verlag, 1981, pp 55-71.

Hand I: Spielen - Glücksspielen - Krankhaftes Spielen («Spielsucht»); in Korczak D (Hrsg): Die betäubte Gesellschaft. Frankfurt/M., Fischer Taschenbuch, 1986, pp 76-98.

Hand I: Pathologisches Spielen und delinquentes Verhalten; in Payk TR (Hrsg): Dissozialität: Psychiatrische und forensische Aspekte. Stuttgart, Schattauer, 1992, pp 97-118.

Hand I: «Zwangs-Spektrum-Störungen» oder «Nichtstoffgebundene Abhängigkeiten»?; in Mundt C, Linden M, Barnett W (Hrsg): Psychotherapie in der Psychiatrie. Wien, Springer, 1997, pp 209-219.

Hand I: Pathologisches Kaufen - Kaufzwang, Kaufrausch oder Kaufsucht?; in Lenz G, Demal U, Bach M (Hrsg): Spektrum der Zwangsstörungen. Wien, Springer, 1998a, pp 123-132. 
Hand I: Pathological gambling - a negative state model and its implications for behavioral treatments. CNS Spectr 1998b;3:58-71.

Hand I: Verhaltenstherapie für Zwangskranke und deren Angehörige; in Möller HJ (ed): Therapie psychiatrischer Erkrankungen, ed 2. Stuttgart, Thieme, 2000, pp 752-772.

Hand I: Multimodale Verhaltenstherapie; in Ahrens S, Schneider W (Hrsg): Lehrbuch der Psychotherapie und psychosomatischen Medizin, ed 2. Stuttgart, Schattauer, 2002a, pp 560-569.

Hand I: Systemische Aspekte in der Verhaltenstherapie von Zwangsstörungen; in Ecker W (ed): Die Behandlung von Zwängen. Bern, Huber, 2002b, pp 81 100

Hand I, Kaunisto E: Multimodale Verhaltenstherapie bei problematischem Verhalten in Glücksspielsituationen («Spielsucht»). Suchtgefahren 1984;30:1-11.

Harvard Medical School Division on Addictions. The Institute for Research on Pathological Gambling and Related Disorders: www.hms.harvard.edu/doa/institute. Holden C: 'Behavioral' addictions: Do they exist? Science 2001;294. www. sciencemag.org.

Hollander E (ed): Obsessive-Compulsive Related Disorders. Washington, DC, American Psychiatric Press, 1993.

Ibáñez-Cuadrado A, Blanco-Jerz C, Moreyra P, SaizRuiz J: Gender differences in pathological gambling. American Psychiatric Association, Annual meeting, May 5-10, 2001, New Orleans, Poster Nr 671.

Jacobs DF: A general theory of addictions - application to the theory and rehabilitation planning for pathological gamblers; in Galski T (ed): The Handbook of Pathological Gambling. Springfield, Charles C Thomas, 1987, pp 169-194.

Jacobs DF: A general theory of addictions: Rational for and evidence supporting a new approach for understanding and treating addictive behaviors; in Shaffer HJ, Stein SA, Gambino B, Cummings TN (eds): Compulsive Gambling: Theory, Research and Practice. Lexington, MA, Lexington Books, 1989, pp 35-64. Jacobs D: Incidence of childhood trauma among pathological gamblers: Implications for theory and practice. 12. International Conference on Gambling and Risk Taking, Vancouver, 2003. Erhältl. unter http:// www.unr.edu/gaming/HMR\%20Order\%20form.pdf Khantzian EJ: The self-medication hypothesis of addictive disorders: A focus on heroin and cocaine. Am J Psychiatry 1985;142:1259-1264.

Khantzian EJ: The self-medication hypothesis of substance use disorders: A reconsideration and recent applications. Harv Rev Psychiatry 1997;4:231-244.

Khantzian EJ: The self-medication hypothesis: Applications to gambling and other behavioral disorders. Kongress «Rethinking Addiction», Las Vegas, 8.10.12.2002, abstr p12.

Kidman R: Reshaping Gambling Beliefs through Group Therapy. www.the wagerorg.current.htm. 2003 (July 16);8:no 29.

Klepsch R, Hand I, Wlatzlo Z, Kaunisto E, Friedrich B: Langzeiteffekte multimodaler Verhaltenstherapie bei krankhaftem Glücksspielen: I Retrospektive Katamnese der Hamburger Pilotstudie. Suchtgefahren 1987:33:137-147

Klepsch R, Hand I, Wlatzlo Z, Friedrich B, Fischer M, Bodek D: Langzeiteffekte multimodaler Verhaltenstherapie bei krankhaftem Glücksspielen III: Zweite Prospektive Katamnese der Hamburger Projektstudie. Suchtgefahren 1989a;35:35-49.

Klepsch R, Hand I, Wlatzlo Z, Kaunisto E, Friedrich B: Pathologisches Spielen; in Hand I, Wittchen HU (Hrsg): Verhaltenstherapie in der Medizin. Berlin, Springer, 1989b, pp 313-326.
Ladouceur R, Sylvain C, Boutin C, Lachance S, Doucet C, Leblond J: Cognitive treatment of pathological gambling. J Nerv Ment Dis 2001;189:766-773. Ladouceur R, Sylvain C, Boutin C, Lachance S, Doucet C, Leblond J: Group therapy for pathological gamblers: A cognitive approach. Behav Res Ther 2003;41:587-596.

Lesieur HR: The Chase: Career of the Compulsive Gambler. Cambridge MA, Schenkman, 1984.

Lesieur HR: The female pathological gambler; in Eadington WR (ed): Gambling Research: Proceedings of the Seventh International Conference on Gambling and Risk Taking, vol 5. Reno, University of Nevada, 1988, pp 230-258

Lumley MA, Roby KJ: Alexithymia and pathological gambling. Psychother Psychosom 1995;63:201-216.

Madras BK: The brain and addiction: Neurobiological perspectives on drugs, gambling and other behavioral disorders. Konferenz «Rethinking Addiction», Las Vegas, 8-10.12.2002, abstr p 9.

Matussek P: Zwang und Sucht. Nervenarzt 1958; 9: 452-456.

McCormick RA, Taber JI, Kruedelbach N, Russo A: Personality profiles of hospitalized pathological gamblers. J Clin Psychol 1987;43:521-527.

McCormick RA, Taber JI, Kruedelbach N: The relationship between attributional style and post-traumatic stress disorder in addicted patients. J Trauma Stress 1989:2:477-487.

Meyer G, Bachmann M: Spielsucht - Ursachen und Therapie. Berlin, Springer, 2000.

Milkman H, Sunderwirth S: Craving for Ecstasy - the Consciousness and Chemistry of Escape. New York, John Wiley and Sons, 1998.

Miller WR, Rollnick S: Motivational Interviewing Preparing People to Change Addictive Behaviors. New York, Guilford, 1991.

Moran E: Varieties of pathological gambling. Brit $\mathbf{J}$ Psychiatry 1970;160:593-597.

Neudecker A, Rufer M: Ambulante Verhaltenstherapie bei Trichotillomanie: Überblick, Störungsmodell und Fallbeispiel. Verhaltenstherapie 2004;14:90-98

Niederland WG: A contribution to the psychology of gambling. Psychoanal Forum 1967;2:175-179.

Niederland WG: Compulsive gambling and the «surviver syndrom». Am J Psychiatry 1984;141:1013.

O'Connor J, Ashenden R, Raven M, Allsop St: Current «Best Practice» Interventions for Gambling Problems: A Theoretical and Empirical Review. National Center for Education and Training on Addiction (NCETA), Flinders University of SA, 2004, www.problemgambling.vic.gov.au.

Orford J: Excessive Appetites - a Psychological View of Addictions. New York, John Wiley und Sons, 1985. Ottinger PW: The early warning system that failed: A personal account. J Gambling Behav 1988;4:309-311.

Petry J: Glücksspielsucht - Entstehung, Diagnostik und Behandlung. Göttingen, Hogrefe, 2003.

Petry NM: How treatments for pathological gambling can be informed by treatments for substance use disorders. Exp Clin Psychopharmacol 2002a;10:184-192. Petry NM: The efficacy of cognitive-behavioral therapy for treatment of pathological gambling. www.aaap.org/meetings/2002am/Proceedings/2002symposium 2.html (b)

Prochaska JO, Velicer WF, Rossi JS, Goldstein MG, Marcus BH, Fiore C, Harlow L, Redding CA, Rosenbloom D, Rossi SR: Stages of change and decisional balance for twelve problem behaviors. Health Psychol 1994;13:39-46.
Prochaska JO: How do people change, and how can we change to help many more people?; in Hubble MA, Duncan BC, Miller SD (eds): The Heart and Soul of Change. Washington DC, American Psychological Association Press, 1999.

Prochaska JO: The natural history of addictions stages of change for gambling and other behaviora disorders. Konferenz «Rethinking Addiction», Las Vegas, 8-10.12.2002, abstr p 8.

Rosenthal RJ: The role of medication in the treatment of pathological gambling: Bridging the gap between research and practice. www.camh.net/egambling/issue10 (2004)/ejgi 10 rosenthal.html.

Sauke G: Kleptomanie: Überblick zum Forschungsstand und Ergebnisse einer verhaltenstherapeutischen Studie. Verhaltenstherapie 2004;14:100-110.

Schumacher W: Die Beurteilung der Schuldfähigkei bei nicht-stoffgebundenen Abhängigkeiten (Spielleidenschaft, Fetischismen, Hörigkeit); in Hamm R (Hrsg): Festschrift für Werner Sarstedt. Berlin, de Gruyter, 1981, pp 361-372.

Shaffer HJ: Strange bedfellows: A critical view of pathological gambling and addiction. Addiction 1999; 94:1445-1448.

Shaffer HJ: Rethinking addiction - how gambling and other behavioral addictions are changing the concept and treatment of alcohol and substance use disorders. Konferenz «Rethinking Addiction», Las Vegas, 8-10.12.2002, abstr pp 4-6.

Shaffer HJ: A critical view of pathological gambling and addiction - comorbidity makes for syndromes and other strange bedfellows; in Reich G (ed): Gambling: Who Wins? Who Loses? New York, Prometheus Books, 2003, pp175-190.

Shaffer HJ, Hall MN: Updating and refining metaanalytic prevalence estimates of disordered gambling behavior in the United States and Canada. Can J Pub Health 2001;92:168-172.

Sylvain C, Ladouceur R, Boisvert J: Cognitive and behavioral treatment of pathological gambling - a controlled study. J Consult Clin Psychol 1997;65:727-732. Taber JI, McCormick RA, Ramirez LF: The prevalence and impact of major life stressors among pathological gamblers. Int J Addict 1987;22:71-79.

Tavares H, Zilberman ML, Beites FJ, Gentil V: Pathological gambling in women; in: Proceedings of the American Psychiatric Association Annual meeting. New Orleans, APA, 2001, p 151.

Tavares H, Zilberman M, el-Guebaly N: Temperament and craving - do gamblers crave as alcoholics? Poster presentation. Konferenz «Rethinking Addiction», Las Vegas, 8-10.12.2002, abstr p 28.

The Wager: Why people gamble. The Wager: The weekly addiction gambling educational report 1998;3 (11). www.thewager.org/

The Wager: Stress Anxiety \& Why gamblers gamble 2000;5(27).www.thewager.org.

Tyndel M: Gambling - an addiction. Addictions 1963 10:40-48.

Vent P: Spielsucht als Affektregulation. Stuttgart, Klett-Cotta, 1999.

Wildman II R: Gambling - an Attempt at an Integration. Edmonton, Alberta, Wynne Resources, 1997.

Wlazlo Z, Hand I, Klepsch R, Friedrich B, Fischer M: Langzeiteffekte multimodaler Verhaltenstherapie bei krankhaftem Glücksspielen. II: Prospektive Katamnese der Hamburger Projektstudie. Suchtgefahren 1987;33:148-161.

Zimmerman MA, Meeland T, Krug SE: Measurement and structure of pathological gambling behavior. J Pers Assess 1985;49:76-81. 